\title{
ЭКОНОМИЧЕСКИЙ РОСТ: АНАЛИЗ ПЕРЕХОДА В РАВНОВЕСНОЕ СОСТОЯНИЕ
}

\author{
В. Р. Абдуллин, Р. З. Абдуллин \\ Байкальский государственный университет, г. Иркутск, Российская Федерация
}

Информация о статье

Дата поступления

19 марта 2018 г.

Дата принятия к печати

21 мая 2018 г.

Дата онлайн-размещения 8 июня 2018 г.

\section{Ключевые слова}

Экономический рост; дискретная модель Солоу; стационарное решение; переходный процесс

\begin{abstract}
Аннотация
Для модели экономического роста Солоу с дискретным временем приведено условие существования единственного притягивающего ненулевого стационарного решения. Исследовано влияние годового темпа роста числа занятых, доли годового выбытия капитала и нормы накопления на стационарное решение. Получены нижняя и верхняя оценки отклонения капиталовооруженности от стационарного значения. За время переходного процесса принято время уменьшения начального отклонения капиталовооруженности от стационарного значения в десять раз. Для различных начальных значений капиталовооруженности получены нижняя и верхняя оценки времени переходного процесса. Проведен анализ влияния параметров модели на оценки времени переходного процесса. Для модели Солоу с производственной функцией Кобба - Дугласа с постоянной отдачей от масштаба производства эти оценки не зависят от нормы накопления. Для реалистических значений параметров модели с производственной функцией Кобба - Дугласа приведены числовые значения оценок времени переходного процесса.
\end{abstract}

\section{ECONOMIC GROWTH: ANALYSIS OF TRANSITION TO EQUILIBRIUM STATE}

\author{
Vladimir R. Abdullin, Rafael Z. Abdullin \\ Baikal State University, Irkutsk, the Russian Federation
}

\section{Article info}

Received

March 19, 2018

Accepted

May 21, 2018

Available online

June 8, 2018

\section{Keywords}

Economic growth; Solow model in discrete time; Stationary solution; Transition

\begin{abstract}
The Solow model in discrete time is set with the existence condition of the unambiguous non-zero stationary attractor solution. The influence of the annual growth rate of employment, the share of annual capital disposal and the rate of accumulation for stationary solution are investigated. The lower and upper deviation estimates of capital endowment ratios from stationary value are considered. The transient time is considered as the time of initial displacement reduction. The lower and upper estimates of the transient time are obtained for the different initial values of the capital endowment ratios. The analysis of the influence of model parameters on the transient process time estimations is carried out. For the Solow model with the CobbDouglas production function with the trendless decreasing return of scale, these estimates do not depend on the rate of accumulation. The numerical values of estimated transient time are given for the feasible parameter values of the Cobb-Douglas production function model.
\end{abstract}

В модели экономического роста Солоу [1-3] с непрерывным временем изменение во времени ее переменных обусловлено включением в нее автономного обыкновенного дифференциального уравнения первого порядка для капитала. Модель Солоу с 
непрерывным временем достаточно хорошо изучена. Для непрерывной модели Солоу, включающей эндогенный технический прогресс, в [2] приведен анализ влияния на стационарное состояние темпов роста населения и технического прогресса, норм накопления и выбытия капитала. Анализ переходного процесса к стационарному состоянию в непрерывной модели Солоу с производственной функцией Кобба - Дугласа содержится в [3]. В этой же работе для учета запаздывания в освоении инвестиций в непрерывную модель Солоу введено дополнительное диффреренциальное уравнение для ввода основных фоондов. Для расширенной таким образом модели показано существование ненулевого притягивающего стационарного решения и определена норма накопления, обеспечивающая максимальное потребление на траектории сбалансированного роста. Устойчивость непрерывной модели Солоу с кусочно-постоянным запаздыванием в освоении инвестиций исследована в [4]. В [5] для непрерывной модели Солоу с производственной функцией, учитывающей разделение капитала на основной и оборотный, поставлена и решена задача о существовании и единственности оптимального роста. Непрерывные модели экономического роста с изменяющимися во времени параметрами исследованы в [6].

Модель Солоу с дискретным временем, в которой динамика капитала описывается нелинейным разностным уравнением первого порядка $[7 ; 8]$, менее изучена. В работе [9] для модификации дискретной модели Солоу с разбиением капитала на основной и оборотный получено нелинейное разностное уравнение для структуры капитала - оснащенности основного капитала оборотным, доказано существование, единственность и устойчивость стационарного решения этого уравнения. Для модификации дискретной модели Солоу, учитывающей запаздывание в освоении инвестиций путем их фрормирования из продукции текущего и предыдущего периода, в [10] получены условия существования, единственности и притяжения стационарного режима.

Следует отметить, что значения параметров модели Солоу с дискретным временем достаточно просто определяются по статистическим данным как среднегодовой темп прироста числа занятых, среднегодовая доля выбытия капитала и средняя норма накопления. Параметры же непрерывной модели представляют подлежащие нахождению по статистическим данным мгновенные темпь прироста населения и капитала. По сравнению с моделью с непрерывным временем модель с дискретным временем значительно упрощает анализ влияния запаздывания в освоении инвестиций на экономический рост.

В дискретном варианте модели Солоу капиталовооруженность текущего периода связывается с капиталовооруженностью предыдущего периода нелинейным разностным уравнением. В связи с этим возникает вопрос о существовании и единственности решения этого уравнения. В модели Солоу как с непрерывным, так и с дискретным временем стационарное состояние (режим) определяется в предположении постоянства ее параметров. Условие существования ненулевого притягивающего стационарного решения и смещение этого решения, вызванное изменением параметров непрерывного варианта модели, приведены, например, в [2; 3]. Для дискретного варианта модели эти вопросы не рассмотрены.

В непрерывной и дискретной модели Солоу основное внимание уделяется характеристикам изменения экономических показателей в стационарном режиме. Решения непрерывной и дискретной модели сходятся к стационарному режиму при неограниченном возрастании времени, т. е. когда время стремится к бесконечности. Предположение ○ постоянстве параметров модели будет довольно жестким, если решения модели недостаточно быстро приближаются к стационарному состоянию. Поэтому представляет интерес время переходного процесса, например десятикратного уменьшения начального отклонения от стационарного состояния, нижняя и верхняя оценки этого времени, зависимость этих оценок от параметров модели.

Модель экономического роста Солоу [1-3] с дискретным временем $t$, измеряемым в годах, $t=0,1,2, \ldots$, описывается соотношениями

$$
\begin{gathered}
X(t)=F(K(t), L(t)), \\
L(t)=(1+\gamma) \cdot L(t-1), \quad L(0)=L_{0}, \\
K(t)=(1-\mu) \cdot K(t-1)+I(t), K(0)=K_{0}, \\
I(t)=\rho X(t), \\
C(t)=(1-\rho) X(t) .
\end{gathered}
$$

Здесь и далее $X(t)$ - валовый внутренний продукт (ВВП) года $t ; F(K(t), L(t))-$ линейно однородная производственная функция С положительными частными производными первого порядка и отрицательными частными производными второго порядка; $L(t)-$ численность занятых в году $t ; \gamma-$ годовой темп роста числа занятых; $K(t)$ - капитал 
(производственные фронды) года $t ; \mu-$ доля годового выбытия капитала; $I(t)$ - инвестиции года $t$ в капитал; $\rho-$ норма накопления; $C(t)$ - непроизводственное потребление в году $t$. Параметры модели - постоянные величины, удовлетворяющие ограничениям $0<\gamma<1,0<\mu<1,0<\rho<1$.

В удельных показателях (капиталовооруженности $k(t)=\frac{K(t)}{L(t)}$ на одного занятого, ВВП на одного занятого $x(t)=\frac{X(t)}{L(t)}=F\left(\frac{K(t)}{L(t)} ; 1\right)=f(k(t))$, инвестициях на одного занятого $i(t)=\frac{l(t)}{L(t)}=$ $=\rho \cdot x(t)$, потребления на одного занятого $\left.c(t)=\frac{C(t)}{L(t)}\right)$ модель (1) примет вид

$$
\begin{gathered}
x(t)=f(k(t)) \\
k(t)=\frac{1-\mu}{1+\gamma} k(t-1)+\rho \cdot f(k(t)) \\
i(t)=\rho \cdot f(k(t)) \\
c(t)=(1-\rho) f(k(t))
\end{gathered}
$$

где $f(0)=0, f^{\prime}(k)>0, f^{\prime \prime}(k)<0$. В этой модели капиталовооруженность $k(t)$ определяется как неявная функция ее предшествующего значения $k(t-1)$ из нелинейного разностного уравнения первого порядка. Покажем, что это уравнение имеет решение, и притом единственное. Для этого представим второе уравнение системы (2) в виде

$$
k(t)-\rho \cdot f(k(t))=\frac{1-\mu}{1+\gamma} k(t-1) .
$$

Правая часть этого уравнения при заданном $k(t-1)>0$ положительна.

Если $\rho \cdot f(k)<k$ при любых $k>0$, то из условий $f(0)=0, f^{\prime}(k)>0, f^{\prime \prime}(k)<0$ вытекает неравенство $\rho \cdot f^{\prime}(0)<1$, так как в противном случае при значениях $k$, близких к нулю, выполнялось бы неравенство $k<\rho \cdot f(k)$. Отсюда получаем $1-\rho \cdot f^{\prime}(k)>0$ при $k>0$. Следовательно, в этом случае функция $k-\rho \cdot f(\mathrm{k})$ неограниченно возрастает и достигает значения $\frac{1-\mu}{1+\gamma} k(t-1)$ при некотором положительном $k$, которое и является единственным искомым решением $k(t)$ второго уравнения системы (2).

Если уравнение $k-\rho \cdot f(k)=0$ имеет положительное решение $k_{0}$, то в силу $f(0)=0$, $f^{\prime}(k)>0$ и $f^{\prime \prime}(k)<0$ имеем $k-\rho \cdot f(k)<0$ при $0<k<k_{0}$ и $k-\rho \cdot f(k)>0$ при $k>k_{0}$. В точке $k_{0}$ угловой коэффрициент прямой $y=k$ больше углового коэффициента кривой $y=\rho \cdot f(k)$, т. е. $\rho \cdot f^{\prime}\left(k_{0}\right)<1$. Следовательно, $1-\rho \cdot f^{\prime}(k)>0$ при $k>k_{0}$. Значит, и в этом случае при $k>k_{0}$ функция $k-\rho \cdot f(k)$ неограниченно возрастает и достигает значения $\frac{1-\mu}{1+\gamma} k(t-1)$. при некотором $k$, которое и является единственным искомым решением $k(t)$ второго уравнения системы (2).

Стационарный режим, при котором все удельные показатели остаются неизменными во времени, определяется стационарным значением $k^{*}$ капиталовооруженности. Согласно второму уравнению системы (2), стационарное значение $k^{*}$ капиталовооруженности находится как решение уравнения

$$
\frac{\gamma+\mu}{1+\gamma} \cdot k^{*}=\rho \cdot f\left(k^{*}\right) .
$$

Tеорема 1. При $f(0)=0, f^{\prime}(k)>0, f^{\prime \prime}(k)<0$, постоянных значениях параметров $\gamma, \mu, \rho$ и выполнении условия

$$
\frac{\gamma+\mu}{1+\gamma}<\rho \cdot f^{\prime}(0)
$$

уравнение (3) имеет единственное ненулевое стационарное решение $k^{*}$, к которому при $t \rightarrow \infty$ сходятся все решения $k(t)$ второго уравнения системы (2) с $k(0) \neq k^{*}$. При этом для $k(0)<k^{*}$ решения $k(t)$ сходятся к $k^{*}$, возрастая, а при $k(0)>k^{*}$, убывая.

Доказательство. Согласно условию (4), в точке $k=0$ угловой коэффрициент прямой $y=\frac{\gamma+\mu}{1+\gamma} \cdot k$ меньше углового коэфорициента касательной к графику функции $\rho \cdot f(k)$, который в силу $\rho \cdot f^{\prime \prime}(k)$ уменьшается с увеличением k. Следовательно, существует единственное ненулевое решение $k^{*}$ уравнения (3) и для $k<k^{*}$ выполняется неравенство $\frac{\gamma+\mu}{1+\gamma} \cdot k<\rho \cdot f(k)$, а для $k>k^{*}-$ неравенство $\frac{\gamma+\mu}{1+\gamma} \cdot k>\rho \cdot f(k)$. Отсюда также следует существование точки $\tilde{k}>k^{*}$, в которой угловой коэфффициент касательной к графрику функции $\rho \cdot f(k)$ совпадает с угловым коэфффициентом прямой $y=\frac{\gamma+\mu}{1+\gamma} \cdot k$, т. е. $\rho \cdot f^{\prime}(\tilde{k})=\frac{\gamma+\mu}{1+\gamma}$, и при $k>\tilde{k}$ выполняются неравенства $\rho \cdot f^{\prime}(k)<$

$$
\begin{aligned}
<\frac{\gamma+\mu}{1+\gamma} & <1,1-\rho \cdot f^{\prime}(k)>\frac{1-\mu}{1+\gamma}>0 n \\
0 & <\frac{1-\mu}{(1+\gamma)\left(1-\rho \cdot f^{\prime}(k)\right)}<1 .
\end{aligned}
$$

Для отклонения $k(t)-k^{*}$ решения $k(t)$ от стационарного значения $k^{*}$ из (2) и (3), используя формулу конечных приращений, получаем: 


$$
\begin{aligned}
& k(t)-k^{*}=\frac{1-\mu}{1+\gamma} k(t-1)+\rho \cdot f(k(t))-k^{*}= \\
= & \frac{1-\mu}{1+\gamma}\left(k(t-1)-k^{*}\right)+\rho \cdot f(k(t))-\frac{\mu+\gamma}{1+\gamma} \cdot k^{*}= \\
= & \frac{1-\mu}{1+\gamma}\left(k(t-1)-k^{*}\right)+\rho \cdot f(k(t))-\rho \cdot f\left(k^{*}\right)= \\
= & \left.\frac{1-\mu}{1+\gamma}\left(k(t-1)-k^{*}\right)+\rho \cdot f^{\prime}\left(k_{l}\right)(k(t))-k^{*}\right),
\end{aligned}
$$

где $k_{1}$ - некоторое значение между $k(t) n k$. Таким образом, имеем

$$
k(t)-k^{*}=\frac{1-\mu}{(1+\gamma)\left(1-\rho \cdot f^{\prime}\left(k_{l}\right)\right)}\left(k(t-1)-k^{*}\right) .
$$

Выше было показано, что при $k(0)>0$ решение $k(t)$ положительно при любых $t>0$. В силу этого из второго уравнения системы (2) при любых $t>0$ следует $k(t)>\rho \cdot f(k(t))$. Отсюда и условий $f(0)=0, f^{\prime}(k)>0, f^{\prime \prime}(k)<0$ вытекает неравенство $\rho \cdot f^{\prime}(0)<1$, так как в противном случае при значениях $k$, близких к нулю, выполнялось бы неравенство $k<\rho \cdot f(k)$. Из $\rho \cdot f^{\prime}(0)<1$ получаем $1-\rho \cdot f^{\prime}(k)>0$ при $k>0$ и $\frac{1-\mu}{(1+\gamma)\left(1-\rho \cdot f^{\prime}\left(k_{1}\right)\right)}>0$.

При $0<k(0)<k^{*}$ из (6) следует $k(1)<k^{*}$, $k(2)<k^{*}, \ldots, k(t)<k^{*}, \ldots$. . Представим приращение капиталовооруженности $\Delta k(t)=k(t)-k(t-1)$ в виде

$$
=\frac{\gamma+\mu}{1+\gamma}(k(t)-k(t-1))-\frac{\gamma+\mu}{1+\gamma} k(t)+\rho \cdot f(k(t)) .
$$

Отсюда, учитывая $k(t)<k^{*}$ и $\rho \cdot f(k(t))-$ $-\frac{\gamma+\mu}{1+\gamma} k(t)>0$, получаем

$$
\Delta k(t)=\left(\frac{1+\gamma}{1-\mu}\right)\left(\rho \cdot f(k(t))-\frac{\gamma+\mu}{1+\gamma} k(t)\right)>0,
$$$$
\text { T. e. } k(t)>k(t-1) \text {. }
$$

Таким образом, при $0<k(0)<k^{*}$ решения $k(t)$ возрастают, оставаясь меньше $k^{*}$. Следовательно, существует предел $\lim k(t)$, который определяется из второго уравнения системы (2), а именно

$$
\lim _{t \rightarrow+\infty} k(t)=\frac{1-\mu}{1+\gamma} \lim _{t \rightarrow+\infty} k(t)+\rho \cdot f\left(\lim _{t \rightarrow+\infty} k(t)\right) .
$$

Это уравнение совпадает с уравнением (3), поэтому $\lim _{t \rightarrow+\infty} k(t)=k^{*}$.

При начальном условии $k(0)>k^{*}$ из (6) и (5) для $t=1$ следует

$$
k(1)-k^{*}<k(0)-k^{*} n k(1)-k^{*}>0 .
$$

Для $t=2$, принимая за начальное значение $k(1)$, из (6) и (5) также получаем $k(2)-k^{*}<k(1)-k^{*}$ n $k(2)-k^{*}>0$. Повторяя это для последующих моментов времени, получаем, что при $k(0)>k^{*}$ решения $k(t)$ убывают, оставаясь больше $k^{*}$. Следовательно, существует предел $\lim k(t)$, который определяется из уравнения (7) и совпадает с $k^{*}$. Что и завершает доказательство теоремы 1.

Из уравнения (3), определяющего стационарное значение капиталовооруженности, следует, что с ростом доли $\mu$ годового выбытия фондов и темпа $\gamma$ прироста занятых угловой коэфрфициент прямой $y=\frac{\gamma+\mu}{1+\gamma} \cdot k$ возрастает, а стационарное значение капиталовооруженности уменьшается. С ростом нормы накопления $\rho$ стационарное значение капиталовооруженности увеличивается. Если в момент времени изменения параметров модели значение капиталовооруженности находится между предыдущим и новым стационарным значениями, то характер движения системы (2) к стационарному состоянию меняется с возрастания на убывание или с убывания на возрастание.

В предположении постоянства параметров модели теорема 1 говорит о сходимости капиталовооруженности $k(t)$ к стационарному значению $k^{*}$ при неограниченном возрастании времени, т. е. при $\dagger \rightarrow+\infty$. Представляет интерес оценка времени переходного процесса, за которое примем время уменьшения начального отклонения капиталовооруженности от стационарного значения на порядок, т. е. времени $t_{p^{\prime}}$ начиная с которого выполняется неравенство

$$
\left|k(t)-k^{*}\right|<0,1 \cdot\left|k(0)-k^{*}\right| .
$$

Теорема 2. При $k(0)>k^{*}$ нижней оценкой времени переходного процесса является целая часть величины

$$
\frac{1}{\lg (1+\gamma)+\lg \left(1-\rho \cdot f^{\prime}(k(0))\right)-\lg (1-\mu)}
$$

а верхней оценкой - целая часть величины

$$
\frac{1}{\lg (1+\gamma)+\lg \left(1-\rho \cdot f^{\prime}\left(k^{*}\right)\right)-\lg (1-\mu)}+1 .
$$

Для $\tilde{k}<k(0)<k^{*}$ нижней оценкой времени переходного процесса является целая часть величины

$$
\frac{1}{\lg (1+\gamma)+\lg \left(1-\rho \cdot f^{\prime}\left(k^{*}\right)\right)-\lg (1-\mu)}
$$

а верхней оценкой - целая часть величины 


$$
\frac{1}{\lg (1+\gamma)+\lg \left(1-\rho \cdot f^{\prime}(k(0))\right)-\lg (1-\mu)}+1
$$

Доказательство. Для отклонения $z(t)=$ $=k(t)-k^{*}$ решения $k(t)$ от стационарного значения $k^{*}$ при доказательстве теоремы 1 было получено (см. фрормулу (6))

$$
z(t)=\frac{1-\mu}{1+\gamma} z(t-1)+\rho f^{\prime}\left(k_{l}\right) z(t),
$$

где $k$ - некоторое значение между $k^{*} n k(t)$.

При $k(0)>k^{*}$ в силу теоремы 1 для любого $t>0$ выполняются неравенства $k^{*}<k(t)<k(0)$, $z(t)>0, f^{\prime}(k(0))<f^{\prime}\left(k_{1}\right)<f^{\prime}\left(k^{*}\right)$. Отсюда и $(13)$ следует

$$
\begin{gathered}
\frac{1-\mu}{1+\gamma} z(t-1)+\rho f^{\prime}(k(0)) z(t)<z(t)< \\
<\frac{1-\mu}{1+\gamma} z(t-1)+\rho f^{\prime}\left(k^{*}\right) z(t) .
\end{gathered}
$$

Учитывая, что для $k>\tilde{k}$ справедливо неравенство $1-\rho \cdot f^{\prime}(k)>0$, из (14) при $k(0)>k^{*}$ получаем

$$
\begin{gathered}
\frac{1-\mu}{(1+\gamma)\left(1-\rho f^{\prime}(k(0))\right)} z(t-1)<z(t)< \\
<\frac{1-\mu}{(1+\gamma)\left(1-\rho f^{\prime}\left(k^{*}\right)\right)} z(t-1) .
\end{gathered}
$$

Отсюда и неравенства (5) следует оценка

$$
\begin{gathered}
\left(\frac{1-\mu}{(1+\gamma)\left(1-\rho f^{\prime}(k(0))\right)}\right)^{t} z(0)<z(t)< \\
<\left(\frac{1-\mu}{(1+\gamma)\left(1-\rho f^{\prime}\left(k^{*}\right)\right)}\right)^{t} z(0),
\end{gathered}
$$

справедливая для любых $t>0$ при условии $k(0)>k^{*}$.

При $\tilde{k}<k(0)<k^{*}$ для любых $t>0$ выполняются неравенства $k(0)<k(t)<k^{*}, z(t)<0$, $f^{\prime}\left(k^{*}\right)<f^{\prime}\left(k_{1}\right)<f^{\prime}(k(0))$ и (5). Следовательно, в этом случае для $z(t)$ также выполняются неравенства (14) и (15). Учитывая, что $z(t-1)<0$, неравенство (15) представим в виде

$$
\begin{gathered}
\frac{1-\mu}{(1+\gamma)\left(1-\rho f^{\prime}(k(0))\right)}>\frac{z(t)}{z(t-1)}> \\
>\frac{1-\mu}{(1+\gamma)\left(1-\rho f^{\prime}\left(k^{*}\right)\right)} .
\end{gathered}
$$

Расписав это неравенство для моментов времени $1,2,3, \ldots, t$ и перемножив их, получаем

$$
\begin{gathered}
\left(\frac{1-\mu}{(1+\gamma)\left(1-\rho f^{\prime}(k(0))\right)}\right)^{t}>\frac{z(t)}{z(0)}> \\
>\left(\frac{1-\mu}{(1+\gamma)\left(1-\rho f^{\prime}\left(k^{*}\right)\right)}\right)^{t} .
\end{gathered}
$$

Следовательно, и в случае $\tilde{k}<k(0)<k^{*}$ для любых $t>0$ выполняется неравенство (16).

За время переходного процесса рассматривается время $t_{p}$ уменьшения на порядок начального отклонения $z(0)=k(0)-k^{*}$ капиталовооруженности от его стационарного значения, т. е. время $t_{p}$, начиная с которого выполняется неравенство $|z(t)|<0,1 \cdot|z(0)|$. Из (16) определим нижнюю и верхнюю оценки времени переходного процесса при начальном значении капиталовооруженности $k(0)>k^{*}$ и $\tilde{k}<k(0)<k^{*}$.

При $k(0)>k^{*}$ отклонение $z(t)>0$ для всех $t \geq 0$ и в силу (5) и (16) условие $|z(t)|<$ $<0,1 \cdot|z(0)|$ выполняется при $t$, удовлетворяющих неравенству

$$
\left(\frac{1-\mu}{(1+\gamma)\left(1-\rho f^{\prime}\left(k^{*}\right)\right)}\right)^{t} \cdot z(0) \leq 0,1 \cdot z(0) .
$$

Логарифмиируя это неравенство по основанию десять, с учетом (5) получаем, что условие $|z(t)|<0,1 \cdot|z(0)|$ выполняется при $t$, удовлетворяющих неравенству

$$
t \geq \frac{1}{\lg (1+\gamma)+\lg \left(1-\rho \cdot f^{\prime}\left(k^{*}\right)\right)-\lg (1-\mu)} .
$$

Таким образом, верхней оценкой времени переходного процесса при $k(0)>k^{*}$ является целая часть величины

$$
\frac{1}{\lg (1+\gamma)+\lg \left(1-\rho \cdot f^{\prime}\left(k^{*}\right)\right)-\lg (1-\mu)}+1 .
$$

Нижняя оценка времени переходного процесса при $k(0)>k^{*}$ определяется в силу оценок (5) и (16) из условия

$$
\left(\frac{1-\mu}{(1+\gamma)\left(1-\rho f^{\prime}(k(0))\right)}\right)^{t} \cdot z(0) \leq 0,1 \cdot z(0) .
$$

Прологарифмировав это неравенство c учетом (5), нижнюю оценку времени переходного процесса получаем как целую часть величины

$$
\frac{1}{\lg (1+\gamma)+\lg \left(1-\rho \cdot f^{\prime}(k(0))\right)-\lg (1-\mu)} .
$$

При $\tilde{k}<k(0)<k^{*}$ отклонение $z(t)<$ $<0$ для всех $t \geq 0$ и в силу (5) и (16) условие $|z(t)|<0,1 \cdot|z(0)|$ выполняется при $t$, удовлетворяющих неравенству

$$
\left(\frac{1-\mu}{(1+\gamma)\left(1-\rho f^{\prime}(k(0))\right)}\right)^{t}|z(0)| \leq 0,1 \cdot|z(0)| .
$$

Логарифмируя это неравенство и учитывая (5), для случая $\tilde{k}<k(0)<k^{*}$ верхнюю оценку времени переходного процесса получаем как целую часть величины 


$$
\frac{1}{\lg (1+\gamma)+\lg \left(1-\rho \cdot f^{\prime}(k(0))\right)-\lg (1-\mu)}+1
$$

Нижняя оценка времени переходного процесса для случая $\tilde{k}<k(0)<k^{*}$ определяется в силу (16) из неравенства

$$
\left(\frac{1-\mu}{(1+\gamma)\left(1-\rho f^{\prime}\left(k^{*}\right)\right)}\right)^{t} \cdot|z(0)| \leq 0,1 \cdot|z(0)|,
$$

которое выполняется при $t$, удовлетворяющих условию

$$
t \geq \frac{1}{\lg (1+\gamma)+\lg \left(1-\rho \cdot f^{\prime}\left(k^{*}\right)\right)-\lg (1-\mu)} .
$$

Следовательно, нижней оценкой времени переходного процесса в этом случае является целая часть величины

$$
\frac{1}{\lg (1+\gamma)+\lg \left(1-\rho \cdot f^{\prime}\left(k^{*}\right)\right)-\lg (1-\mu)},
$$

что и завершает доказательство теоремы 2.

Из выражений (9)-(12) следует, что с ростом доли $\mu$ годового выбытия капитала и темпа $\gamma$ годового прироста занятых верхняя и нижняя оценки времени переходного процесса уменьшаются, а с ростом нормы накопления $\rho$ увеличиваются.

Рассмотрим оценки времени переходного процесса для модели экономического роста (1) с производственной функцией Кобба - Дугласа с постоянной отдачей от масштаба производства

$$
F(K(t), L(t))=A \cdot K(t)^{\alpha} \cdot L(t)^{1-\alpha}, \text { c } 0<\alpha<1 .
$$

В этом случае $f(k(t))=A \cdot k^{\alpha}(t), f^{\prime}(k(t))=$ $=A \cdot \alpha \cdot k^{\alpha-1}(t)$ и $\lim _{k \rightarrow 0+} f^{\prime}(k)=+\infty$, следователь-

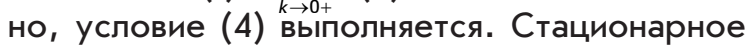
значение $k^{*}$ капиталовооруженности и величины $\tilde{k}$ и $f^{\prime}\left(k^{*}\right)$ определяются как

$$
\begin{gathered}
\dot{k}^{*}=\left(\frac{\rho \cdot \boldsymbol{A} \cdot(1+\gamma)}{\gamma+\mu}\right)^{\frac{1}{1-\alpha}}, \tilde{k}=\left(\frac{\alpha \cdot \rho \cdot \boldsymbol{A} \cdot(1+\gamma)}{\gamma+\mu}\right)^{\frac{1}{1-\alpha}} \\
n f^{\prime}\left(k^{*}\right)=\frac{\alpha \cdot(\gamma+\mu)}{\rho \cdot(1+\gamma)} .
\end{gathered}
$$

Отсюда и (10) для случая $k(0)>k^{*}$ верхняя оценка времени переходного процесса находится как целая часть величины

$$
\frac{1}{\lg (1+\gamma-\alpha \cdot(\gamma+\mu))-\lg (1-\mu)}+1
$$

которая не зависит от нормы накопления $\rho$ и $k(0)$. Для $k(0)=\beta \cdot k^{*}$ с $\beta>1$ имеем $f^{\prime}(k(0))=f^{\prime}\left(\beta \cdot k^{*}\right)=\beta^{\alpha-1} \cdot \frac{\alpha(\gamma+\mu)}{\rho(1+\gamma)}$, и в силу (9) нижняя оценка времени переходного процесса определяется как целая часть величины

$$
\frac{1}{\lg \left(1+\gamma-\beta^{\alpha-1} \cdot \alpha \cdot(\gamma+\mu)\right)-\lg (1-\mu)},
$$

которая также не зависит от нормы накопления $\rho . И з(11)$ для $\tilde{k}<k(0)<k^{*}$ нижняя оценка времени переходного процесса находится как целая часть величины

$$
\frac{1}{\lg (1+\gamma-\alpha \cdot(\gamma+\mu))-\lg (1-\mu)}
$$

которая не зависит от нормы накопления $\rho$ и $k(0)$. Из (12) для начального значения фрондовооруженности $k(0)=\beta \cdot k^{*}$ с $\alpha^{\frac{1}{1-\alpha}}<\beta<1$, что равносильно условию $\tilde{k}<k(0)<k^{*}$, верхняя оценка времени переходного процесса определяется как целая часть величины

$$
\frac{1}{\lg \left(1+\gamma-\beta^{\alpha-1} \cdot \alpha \cdot(\gamma+\mu)\right)-\lg (1-\mu)}+1,
$$

которая также не зависит от нормы накопления $\rho$.

Например, для модели экономического роста (1) с производственной фрункцией Кобба - Дугласа с $\alpha=0,5$, годовым темпом прироста числа занятых $\gamma=0,01$, долей годового выбытия капитала $\mu=0,05$ и $k(0)=1,5 \cdot k^{*}$ нижняя и верхняя оценки времени переходного процесса соответственно составляют 62 года и 75 лет. При увеличении доли годового выбытия капитала до $\mu=0,1$ при неизменных остальных параметрах нижняя оценка времени переходного процесса уменьшается до 32 лет, а верхняя - до 39 лет.

\section{СПИСОК ИСПОЛЬЗОВАННОЙ ЛИТЕРАТУРЫ}

1. Solow R. M. A Contribution to the Theory of Economic Growth / R. M. Solow // The Quarterly Journal of Economics. - 1956. - Vol. 70, № 1. - P. 65-94.

2. Туманова Е. А. Макроэкономика. Элементы продвинутого подхода : учебник / Е. А. Туманова, Н. Л. Шагас. — М. : Инфрра-М, 2004. - 400 с.

3. Колемаев В. А. Математическая экономика : учебник / В. А. Колемаев. - 3-е изд., стер. - М. : ЮнитиДана, 2012. - 401 с.

4. Симонов П. М. Об одном методе исследования динамических моделей макроэкономики / П. М. Симонов / / Вестник Пермского университета. Сер.: Экономика. - 2014. - Вып. 1 (20). - С. $14-27$.

5. Вагин А. А. Исследование модификации модели Солоу / А. А. Вагин, П. М. Симонов // Вестник Тамбовского университета. Сер.: Естественные и технические науки. - 2010. - Т. 15, № 6. - С. 1909-1910. 
6. Лебедев В. В. Математическое моделирование нестационарных экономических процессов / В. В. Лебедев, К. В. Лебедев. - М. : еТест, 2011. - 336 с.

7. Коврижных А. Ю. Дифференциальные и разностные уравнения : учеб. пособие / А. Ю. Коврижных, О. О. Коврижных. - Екатеринбург : Изд-во Урал. ун-та, 2014. - 148 с.

8. Романко В. К. Курс разностных уравнений / В. К. Романко. - М. : Физматлит, 2012. - 200 с.

9. Ущев Ф. А. Модель экономической динамики, учитывающая неоднородность капитала / Ф. А. Ущев, В. П. Чернов / / Финансы и бизнес. - 2007. - № 2. - С. 47-59.

10. Абдуллин В. Р. Анализ устойчивости стационара дискретной модели Солоу с учетом запаздывания освоения инвестиций / В. Р. Абдуллин, Р. З. Абдуллин / / Современные тенденции в социально-экономических и гуманитарных науках: теория и практика : сб. науч. тр. / под науч. ред. Т. Г. Озерниковой, Т. Л. Музычук. Иркутск : Изд-во БГУ, 2017. - С. 273-277.

\section{REFERENCES}

1. Solow R. M. A Contribution to the Theory of Economic Growth. The Quarterly Journal of Economics, 1956, vol. 70, no. 1, pp. 65-94.

2. Tumanova E. A., Shagas N. L. Makroekonomika. Elementy prodvinutogo podkhoda [Macroeconomics. Details of Advanced Approach]. Moscow, Infra-M Publ., 2004. 400 p.

3. Kolemaev V. A. Matematicheskaya ekonomika [Mathematical Economics]. $3^{\text {rd }}$ ed. Moscow, Yuniti-Dana Publ., 2012. $401 \mathrm{p}$.

4. Simonov P. M. On a research method of dynamic models of macroeconomics. Vestnik Permskogo universiteta. Seriya: Ekonomika = Perm university herald. Economy, 2014, vol. 1 (20), pp. 14-27. (In Russian).

5. Vagin A. A., Simonov P. M. Research of modification Solow's model. Vestnik Tambovskogo universiteta. Seriya: Estestvennye i tekhnicheskie nauki = Tambov University Reports. Series: Natural and Technical Sciences, 2010, vol. 15, no. 6, pp. 1909-1910. (In Russian).

6. Lebedev V. V., Lebedev K. V. Matematicheskoe modelirovanie nestatsionarnykh ekonomicheskikh protsessov [Mathematical Modelling of Unstable Economic Processes]. Moscow, eTest Publ., 2011. 336 p.

7. Kovrizhnykh A. Yu., Kovrizhnykh O. O. Differentsial'nye i raznostnye uravneniya [Differential and Difference Equations]. Ekaterinburg, Ural University named after the first President of Russia B. N. Yeltsin Publ., 2014. 148 p.

8. Romanko V. K. Kurs raznostnykh uravnenii [Course on Difference Equations]. Moscow, Fizmatlit Publ., 2012. 200 p.

9. Ushchev F. A., Chernov V. P. Dynamic Economic Model Reflecting the Capital Heterogeneity. Finansy i biznes = Finance and Business, 2007, no. 2, pp. 47-59. (In Russian).

10. Abdullin V. R., Abdullin R. Z. Analysis of the stability of the discrete station models of Solow with the account of liquid development investments. In Ozernikova T. G., Muzychuk T. L. (eds.). Sovremennye tendentsii v sotsial'no-ekonomicheskikh i gumanitarnykh naukakh: teoriya i praktika [Contemporary Trends in Socio-economic Sciences and Liberal Arts: Theory and Practice]. Irkutsk, Baikal State University Publ., 2017, pp. 273-277. (In Russian).

\section{Информация об авторах}

Абдуллин Владимир Рафаэлевич - кандидат фризико-математических наук, доцент кафедры математики и эконометрики, Байкальский государственный университет, 664003, г. Иркутск, ул. Ленина, 11, е-mail: abdullin.vladimir@rambler.ru.

Абдуллин Рафаэль Зинатович - кандидат физико-математических наук, доцент кафедры математики и эконометрики, Байкальский государственный университет, 664003, г. Иркутск, ул. Ленина, 11, e-mail: AbdullinRZ@bgu.ru.

\section{Для цитирования}

Абдуллин В. Р. Экономический рост: анализ перехода в равновесное состояние / В. Р. Абдуллин, Р. З. Абдуллин // Известия Байкальского государственного университета. - 2018. - Т. 28, № 2. - C. 223-229. - DOI: $10.17150 / 2500-$ 2759.2018.28(2).223-229.

\section{Authors}

Vladimir R. Abdullin - Ph.D. in Physics and Mathematics, Associate Professor, Department of Mathematics and Econometrics, Baikal State University, 11 Lenin St., 664003, Irkutsk, the Russian Federation, e-mail: abdullin.vladimir@rambler.ru.

Rafael Z. Abdullin - Ph.D. in Physics and Mathematics, Associate Professor, Department of Mathematics and Econometrics, Baikal State University, 11 Lenin St., 664003, Irkutsk, the Russian Federation, e-mail: AbdullinRZ@bgu.ru.

\section{For citation}

Abdullin V. R., Abdullin R. Z. Economic Growth: Analysis of Transition to Equilibrium State. Izvestiya Baykal' skogo gosudarstvennogo universiteta $=$ Bulletin of Baikal State University, 2018, vol. 28, no. 2, pp. 223229. DOI: $10.17150 / 2500-2759.2018 .28(2) .223-229$. (In Russian). 